\title{
MicroRNA-20a promotes proliferation and invasion by directly targeting early growth response 2 in non-small cell lung carcinoma
}

\author{
LAI WEI $^{1}$ and FENGMING RAN ${ }^{2}$ \\ ${ }^{1}$ Department of Chest Radiotherapy; ${ }^{2}$ First Department of Thoracic Surgery, \\ Hubei Cancer Hospital, Wuhan, Hubei 430079, P.R. China
}

Received April 8, 2016; Accepted July 14, 2017

DOI: $10.3892 / 01.2017 .7299$

\begin{abstract}
MicroRNA-20a (miR-20a) serves a notable role in tumor development and progression; it functions differently in different types of malignant tumor, and its role and mechanism in non-small cell lung carcinoma (NSCLC) remains unclear. In the present study, the effects of miR-20a on the proliferation and invasion of NSCLC cells and the underlying mechanisms behind this were investigated. Reverse transcription-quantitative polymerase chain reaction revealed that the expression level of miR-20a was higher in human NSCLC than in normal tissues. Following this, the effect of miR-20a on the proliferation, apoptosis, migration and invasion of NSCLCA-549 cells was further evaluated. In vitro analysis, including a Cell Counting Kit-8, colony formation and Transwell migration assay, indicated that miR-20a-knockdown inhibited the proliferation, invasion and migration, while promoting the cell apoptosis of the A-549 cells. Early growth response 2 (EGR2) protein and mRNA levels were downregulated or upregulated following the overexpression or knockdown of miR-20a, respectively. Dual-luciferase reporter gene assays implied that EGR2 is a direct target gene of miR-20a. The results of the present study indicated that miR-20a may function as an oncomiR in the development of NSCLC by promoting cell viability and motility. The inhibition of miR-20a could even become a novel therapeutic method for the treatment of NSCLC.
\end{abstract}

\section{Introduction}

Lung cancer is one of the most highly malignant types of cancer; its mortality rate in the USA and China is higher than that of other solid tumors (1). Lung cancer is classified into two types, non-small cell lung cancer (NSCLC) and SCLC,

Correspondence to: Dr Fengming Ran, First Department of Thoracic Surgery, Hubei Cancer Hospital, 116 Zhuodaoquan South Road, Hongshan, Wuhan, Hubei 430079, P.R. China

E-mail: ranfm77@163.com

Key words: non-small cell lung carcinoma, microRNA-20a, A-549, early growth response 2 according to its pathological characteristics. NSCLC accounts for $80-85 \%$ of all lung cancer cases (2). NSCLC has a high mortality and the 5-year survival rate is $<15 \%$ despite improvements in cancer treatment (3). The majority of patients develop an aggressive form of NSCLC at the point of diagnosis, which is often at a late stage of disease and which metastasizes to other organs (4). Thus, there is an urgent requirement for the identification of novel biomarkers for early disease detection and therefore improvements to treatment outcome.

MicroRNAs (miRNAs/miRs) are small ( 22 nucleotides in length), single-stranded, endogenous non-coding RNAs that regulate gene expression by causing mRNA degradation or translational suppression by directly binding to the 3'-untranslated regions (3'-UTRs) of target mRNAs (5-7). miRNAs are implicated in a wide range of important physiological processes (8-10). Evidence indicates that miRNAs act as tumor suppressors or novel oncogenes, according to the roles of their target genes, with aberrant miRNA expression being common in various types of human cancer including NSCLC (11-13).

miR-20a belongs to the miR-17-92 cluster, located in the $13 \mathrm{q} 31.1$ chromosomal region (14). Previous studies have revealed that miR-20a is upregulated in several types of cancer, suggesting its pivotal role in tumorigenesis and progression $(15,16)$. However, in other tumors, including oral squamous cancer (17) and hepatic cancer (18), miR-20a acts as a tumor suppressor. These findings suggest that the functions of miR-20a may differ among different cell types. To date, several miRNAs have been identified as being involved in NSCLC, including miR-30c, miR-4500, miR-193a, miR-4782-3p and miR-138 (19-21). However, to the best of our knowledge, the role of miR-20a in the progression of NSCLC and its underlying mechanism has not yet been investigated.

In the present study, the expression of miR-20a in NSCLC samples and the impact of miR-20a on tumor biological processes was examined. Additionally, early growth response 2 (EGR2) was identified as a direct target of miR-20a in NSCLC cells. The present study indicates that miR-20a may be a novel target for the treatment of NSCLC in the future.

\section{Materials and methods}

Tissue samples. A total of 35 human NSCLC tissues and 35 matched normal tissue samples (located $>5 \mathrm{~cm}$ away from the tumor) were collected from patients ( 24 male cases and 
11 female cases (mean age, 57.68 years; range, 48-65 years) who underwent general thoracic surgery in Hubei Cancer Hospital (Wuhan, China) between February 2012 and July 2013. None of the patients with NSCLC had received radio- or chemo-therapy prior to the surgery and none of the patients had a history of having a tumor. These tissue specimens were snap-frozen in liquid nitrogen and stored at $-80^{\circ} \mathrm{C}$. The present study was approved by the Medical Ethics Committee of Hubei Cancer Hospital and written informed consent for publication was obtained from all patients prior to their participation in the study.

Cell lines and culture. The NSCLCA-549, LTEP-a2, and SPC-A1 cell lines and normal bronchial epithelial cell line $16 \mathrm{HBE}$ were obtained from the Chinese Academy of Sciences (Shanghai, China). The three NSCLC cell lines and 16HBE cells were used to determine the expression of miR-20a, whereas A-549 was also used in further functional analysis. The cells were cultured in Dulbecco's modified Eagle's medium (DMEM; Thermo Fisher Scientific, Inc., Waltham, MA, USA) supplemented with $10 \%$ fetal bovine serum (FBS; Thermo Fisher Scientific, Inc.) and 1\% streptomycin/penicillin at $37^{\circ} \mathrm{C}$ in a humidified incubator with $5 \% \mathrm{CO}_{2}$.

Establishment of A-549 cell lines with knocked down miR-20a. Plasmids containing miR-20a siRNA or negative control were purchased from GenePharma (Shanghai, China). A-549 cells were seeded in a 6-well plate. When cells reached $70-80 \%$ confluency, they were transfected with $20 \mathrm{nM}$ miR-20a siRNA and negative control using riboFECT ${ }^{\mathrm{TM}} \mathrm{CP}$ transfection reagents (RiboBio Co., Ltd., Guangzhou, China) following the manufacturer's instructions. miR-20a expression was upregulated by the transfection of miR-20a mimic using Lipofectamine ${ }^{\circledR} 2000$ (Invitrogen; Thermo Fisher Scientific, Inc.). At $24 \mathrm{~h}$ post transfection, the cells were obtained and used in subsequent analysis. Cells were then harvested and subjected to reverse transcription-quantitative polymerase chain reaction (RT-qPCR) analysis of miR-20a expression.

$R T-q P C R$. Total cellular RNA was isolated from tissues and cell lines using TRIzol reagent (Takara Bio, Inc., Otsu, Japan), then reverse transcribed into cDNA using PrimeScript RT Master Mix (Takara Bio, Inc.) according to the manufacturer's instructions. RT-qPCR was performed to determine the expression of miR-20 using a SYBR-Green Mix kit (Promega Corporation, Madison, WI, USA) according to the manufacturer's protocol. Sequences were as follows: miR-20a mimic sense, 5'-UAAAGUGCUUAUAGUGCAGGUAG-3' and antisense 5'-CUACCUGCACUAUAAGCACUUUA-3'; siRNA forward, 5'-UAA AGUGCUUAUAGUGCAGGUAGTT-3' and reverse, 5'-CUACCUGCACUAUAAGCACUUUATT-3'; siRNA negative control forward, 5'-UUCUCCGAACGUGU CACGUTT-3' and reverse, 5'-ACGUGACACGUUCGG AGAATT-3'. RT-qPCR was performed using the Applied Biosystems 7500 Sequence Detection system (Applied Biosystems; Thermo Fisher Scientific, Inc.) and thermocycling conditions were as follows: Initial denaturation at $95^{\circ} \mathrm{C}$ for $5 \mathrm{~min}$, followed by 40 cycles at $95^{\circ} \mathrm{C}$ for $10 \mathrm{sec}$, and at $62^{\circ} \mathrm{C}$ for 40 sec. U6 was used as an endogenous control and relative quantification of miR-20a expression was evaluated using the
$2^{-\Delta \Delta \mathrm{Cq}}$ method (22). All experiments were performed in triplicate.

Cell proliferation assay. To investigate the effect of miR-20a regulation in NSCLC cells, A-549 cells were seeded into 96-well plates at a density of $3 \times 10^{3}$ and cultured overnight. The transfected cells proliferation was measured using the Cell Counting Kit-8 (CCK-8) assay (Dojindo Molecular Technologies, Inc., Kumamoto, Japan). Briefly, $10 \mu 1$ CCK-8 solution was added into each well and the plates were incubated for another $2 \mathrm{~h}$. The optical density was then measured using a microplate reader (Molecular Devices, LLC, Sunnyvale, CA, USA) at an absorbance of $450 \mathrm{~nm}$. Experiments were performed in triplicate and repeated at least three times independently.

Colony formation assay. A total of $24 \mathrm{~h}$ after transfection with miR-20a siRNA or siRNA negative control, A549 cells were treated with $0.25 \%$ trypsin plus $0.5 \mathrm{mM}$ ethylenediaminetetraacetic acid solution at $37^{\circ} \mathrm{C}$ for $3 \mathrm{~min}$ and seeded in 6 -well plates (500 cells per well) and cultured for 2 weeks. Then, cell colonies were stained with $0.5 \%$ crystal violet for $30 \mathrm{~min}$ at room temperature. The number of colony-forming cells were calculated using an AID iSpot Reader (Autoimmun Diagnostika GmbH, Strassberg, Germany). Three independent experiments were performed.

Cell migration and invasion assay. Transwell chambers with 8 - $\mu$ m pores were obtained from Corning Incorporated (Corning, NY, USA). For the migration assay, the transfected A549 cells were harvested and resuspended in $100 \mu \mathrm{l}$ serum-free medium and then transferred to the upper chambers $\left(2 \times 10^{4}\right.$ cells per well). A total of $600 \mu \mathrm{l}$ medium supplemented with $10 \%$ FBS was added to the lower chamber. After incubation for $24 \mathrm{~h}$, cells that had migrated to the bottom chamber were fixed with methanol for $30 \mathrm{~min}$ at room temperature, stained with crystal violet for $30 \mathrm{~min}$ at $37^{\circ} \mathrm{C}$, and then counted under a light microscope. For the invasion assay, the Transwell membrane was pre-coated with $30 \mu \mathrm{l}$ Matrigel [1:3 mixed with phosphate buffered saline (PBS); BD Biosciences, Franklin Lakes, NJ, USA] and incubated for $48 \mathrm{~h}$, with the remaining experimental procedures identical to the migration assay.

Flow cytometry apoptosis assay. Following transfection, A-549 cells were seeded in $25 \mathrm{~cm}^{2}$ culture flasks at a density of $20 \times 10^{4} /$ well in DMEM, and harvested when they reached $70 \%$ confluence. Then, cells were stained with $5 \mu$ l Annexin V-FITC (BD Biosciences) for $15 \mathrm{~min}$ and $5 \mu \mathrm{l}$ propidium iodide (PI) for another $5 \mathrm{~min}$ at room temperature. Cell cycle was determined using a FACScanto Accuri C6 flow cytometer (BD Biosciences, Franklin Lakes, NJ, USA) and the data was analyzed using CFlow Plus 1.0.264.15 software (BD Biosciences).

Western blot analysis. Following transfection, A-549 cells were harvested and lysed using RIPA Lysis buffer (Beyotime Institute of Biotechnology Co., Ltd., Haimen, China). Protein concentrations were determined using a Bicinchoninic Acid Protein Assay kit (Beyotime Institute of Biotechnology, Beijing, China). Equal amounts (40 $\mu \mathrm{g})$ of protein were separated by $10 \%$ SDS-PAGE and transferred 
onto a polyvinylidenedifluoride membrane with a pore size of $0.45 \mu \mathrm{m}$ (Millipore, Billerica, MA, USA). Following blocking in 5\% skimmed milk in Tris-buffered saline-Tween-20 (TBST) for $1 \mathrm{~h}$ at room temperature, the membranes were incubated with the following rabbit anti-human antibodies: Anti-EGR2 antibody (cat. no. ab108399; Abcam, Cambridge, UK) and anti- $\beta$-actin antibody (cat. no. ab16039; Abcam) at the recommended dilution $(1: 1,000)$ overnight at $4^{\circ} \mathrm{C}$. Subsequent to being washed with TBST, the membranes were further incubated with horseradish peroxidase-conjugated goat anti-rabbit secondary antibodies (1:2,000; cat. no. ab150077; Abcam) for $1 \mathrm{~h}$. Enhanced Chemiluminescence Plus kit (GE Healthcare Bio-Sciences, Pittsburgh, PA, USA) was used to visualized the protein bands according to manufacturer's protocol and the protein expression was quantified using Laboratory Work Image Acquisition and Analysis software version 4.0 (UVP, Inc., Upland, CA, USA). $\beta$-actin was used as a loading control.

Dual luciferase reporter assay. To construct a luciferase reporter vector, the EGR2 3'-UTR fragment containing putative binding sites for miR-20a was amplified using PCR and cloned in the psiCHECK-2 vector (Promega Corporation). Site-directed mutagenesis of the miR-20a seed sequence in the 3'-UTR of EGR2 (Mut) was performed using the QuikChange $^{\mathrm{TM}}$ Site-Directed Mutagenesis kit (Stratagene; Agilent Technologies, Inc., Santa Clara, CA, USA) according to the manufacturer's protocol. Next, $1 \mu \mathrm{g}$ of constructs were co-transfected with $1 \mu \mathrm{g}$ miR-20a precursor or control in A-549 cells. Transfection was performed using Lipofectamine ${ }^{\circledR} 2000$ (Thermo Fisher Scientific, Inc.). At $48 \mathrm{~h}$ after transfection, luciferase activity was measured using the Dual-Luciferase ${ }^{\circledR}$ Reporter Assay system (Promega Corporation) following the manufacturer's instructions. Signals were normalized using the Renilla luminescence of the controls.

Animal work. Female nude (BALB/c-nu) mice (n=9, 4-5 weeks old, 17-20 g) were purchased from Vital River Laboratory Animal Technology Co., Ltd. (Beijing, China). The mice were maintained at a temperature of $18-22^{\circ} \mathrm{C}$ and a humidity of 50-60\% under a 12:12 h light-dark cycle. A total of $5 \times 10^{6}$ A549 cells in $0.2 \mathrm{ml}$ PBS were transfected with miR-20a siRNA and siRNA negative control. The cells at exponential stage were harvested, and were then mixed and injected into the left flanks of the mice. with free access to food and water. Tumor size was measured by caliper every 2 days. Both length (L) and width (W) of the tumor were measured and the tumor size was calculated as $1 / 2 \mathrm{LW}^{2}$. After 20 days, the mice were sacrificed and photographed. Tumors were dissected and weighed. Three animals were included in each group.

Target prediction. Three publicly available databases, including TargetScan (23), microRNA (24), miRDB (25), were used to predict the candidate targets of miR-20a. The common gene, which was simultaneously predicted by these three algorithms, was selected for further analysis.

Statistical analysis. All data are expressed as the mean \pm standard deviation. Differences between two groups were assessed using Fisher's exact test or Student's t-test, while differences among multiple groups were analyzed using one-way analysis of variance followed by Bonferroni's multiple comparisons test. $\mathrm{P}<0.05$ was considered to indicate a statistically significant difference.

\section{Results}

miR-20a is upregulated in patients with NSCLC and in NSCLC cell lines. The expression of miR-20a was detected by RT-qPCR in tumor and adjacent normal tissues of 35 patients. Expression of miR-20a in tumor tissues was significantly higher than that in normal tissues (Fig. 1A). Similarly, all three tested NSCLC cell lines exhibited significantly upregulated miR-20a levels compared with the normal control $16 \mathrm{HBE}$ cell line. These data suggested that miR-20a may serve as a prognostic marker for patients with NSCLC.

Levels of miR-20a in NSCLC cell lines with knocked down miR-20a. A-549 cells were transfected with miR-20a-inhibitor or negative-control plasmids (termed miR-20a siRNA and NC-LV cells, respectively). The expression levels of miR-20a were assessed by RT-qPCR. The expression of miR-20a in the miR-20a siRNA cells was decreased compared with that in the NC-LV cells $(\mathrm{P}<0.05$; Fig. 2).

miR-20 a promotes growth and inhibits apoptosis in NSCLC cells. To assess the biological role of miR-20a in the A-549 cell line, a series of experiments were performed. CCK8 was used to determine the effect of miR-20a on A-549 miR-20a siRNA and NC-LV cells. It was revealed that the proliferation of A-549 decreased significantly in the miR-20a siRNA group (Fig. 3A). To determine the long-term effect of miR-20a on the growth ofA-549 cells, a colony formation assay was performed. Cells transfected with miR-20a inhibitor exhibited lower colony formation than cells transfected with control (Fig. 3B).

To further elucidate the mechanism of miR-20a-mediated cell growth in A-549 cells, flow cytometry analysis of apoptosis was performed. The results revealed that, when compared with the control group, miR-20a-knockdown promoted the apoptosis of A-549 (Fig. 3C).

miR-20a promotes migration and invasion of NSCLC cells in vitro. To further assess the effects of miR-20a on malignant tumor progression and metastasis, migration and invasion assays were used to determine the role served by miR-20a. As shown in Fig. 4, knockdown of miR-20a significantly suppressed the migration and invasion of A-549 cells. Collectively, these results suggested that miR-20a increases the migratory and invasive abilities of A-549 cells.

miR-20a promotes tumorigenicity in vivo. To further demonstrate the role of miR-20a, A-549 cells infected with miR-20a inhibitor and inhibitor negative control plasmids were injected into female nude mice. Tumors derived from miR-20a siRNA A-549 cells grew much more slowly than those of the NC group, and the tumor weight was also significantly less than in the NC group (Fig. 5).

EGR2 is a direct target of miR-20a. It is generally accepted that miRNAs regulate the expression of mRNAs by targeting 
A

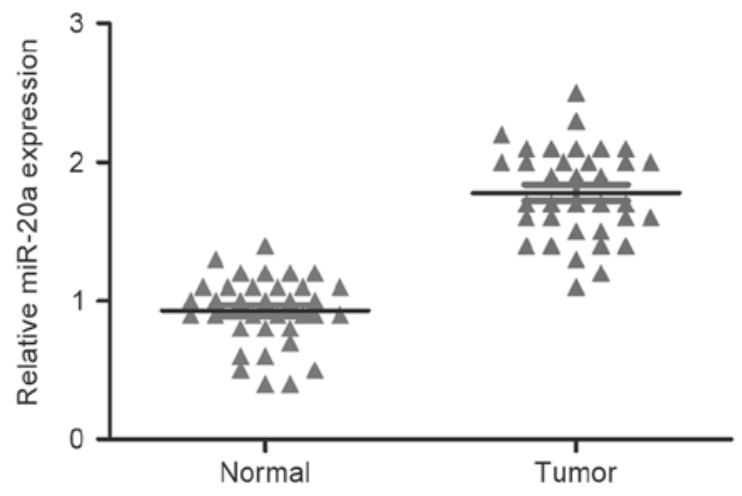

B

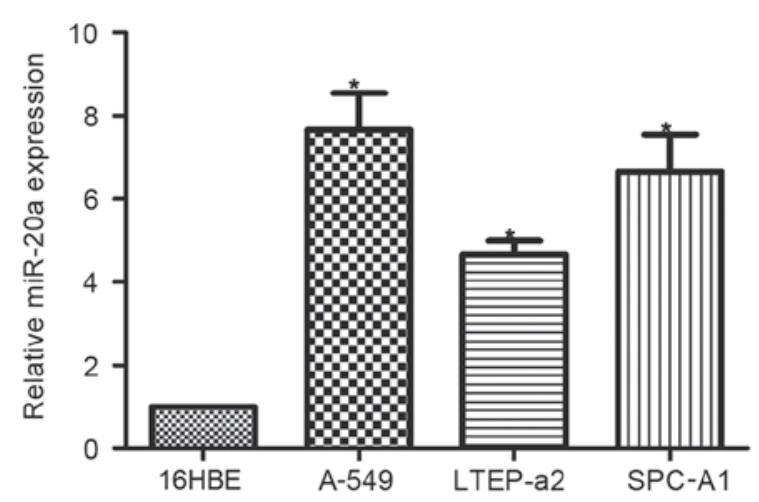

Figure 1. Expression of miR-20a in NSCLC tissues and cell lines. (A) The mRNA expression of miR-20a was assessed by reverse transcription-quantitative polymerase chain reaction using 35 NSCLC tissue samples and matched adjacent non-tumor normal tissues. (B) Average relative expression of miR-20a in three NSCLC cell lines: A-549, LTEP-a2 and SPC-A1. "P<0.05 compared with normal control 16HBE cells. miR-20a, microRNA-20a; NSCLC, non-small cell lung cancer.

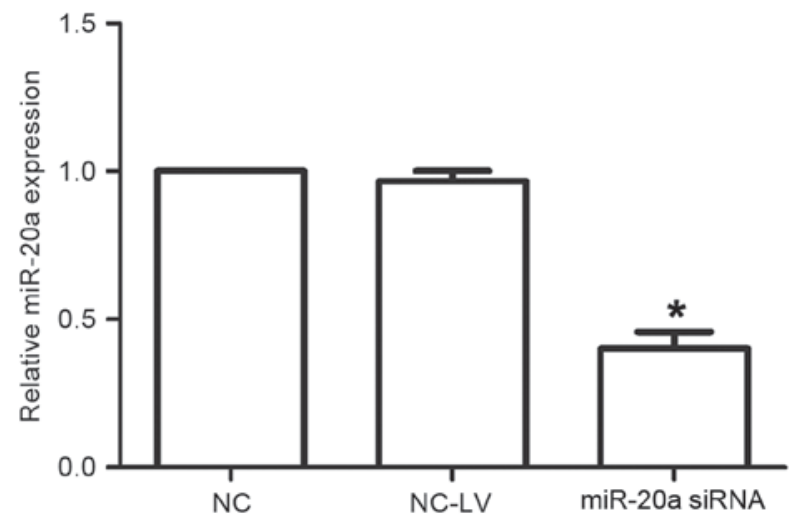

Figure 2. Reduced expression of miR-20a in non-small cell lung cancer cell lines following knockdown. Validation of reduced expression of miR-20a in A-549 cells. * $\mathrm{P}<0.05$ vs. NC-LV. miR-20a, microRNA-20a; NC, negative control; NC-LV, A-549 cells transfected with empty lentivirus negative control; siRNA, short interfering RNA.

the mRNA 3'UTR (26). All of these algorithms (TargetScan, microRNA, miRDB) were indicated that EGR2, a member of a multi-gene family encoding zinc finger proteins that is involved in the regulation of cellular proliferation and the cell cycle, was a putative target of miR-20a. Thus, EGR2 was selected as a target for further analysis (Fig. 6A).

To investigate whether EGR2 was a functional target of miR-20a, a dual-luciferase reporter assay was performed (Fig. 6B). The EGR2 3'-UTR containing the target sequences, or the mutants, was cloned into a luciferase reporter vector. miR-20a suppressed the luciferase activity of the wild-type EGR2 3' UTR, whereas mutation of the miR-20a binding sites blocked this suppression in the A-549 cells. Western blot analysis demonstrated that EGR2 expression was inhibited following transfection of miR-20a precursor in A-549 cells, whereas transfection with the miR-20a inhibitor elevated EGR2 protein levels (Fig. 6C). Similar results were also observed in RT-qPCR analysis, in which the miR-20a precursor decreasedEGR 2 mRNA expression, whereas transfection with the miR-20a inhibitor increased expression of EGR2 mRNA (Fig. 6D). Taken together, these results indicate that miR-20a can directly target EGR2 in A-549 cells.

\section{Discussion}

Although numerous miRNAs are involved in NSCLC carcinogenesis, their underlying molecular mechanisms in NSCLC development remain poorly understood. Hence, it is of great value to investigate the function of miRNAs involved in NSCLC carcinogenesis and to screen for novel targets for diagnosis and therapy. miR-20a and miR-17 (also known as miR-17-5p) are located together in the miR-17-92 cluster, sharing the same seed sequence, AAAGUG (14). Aberrant expression of miR-20a has been observed in colorectal, cervical and prostate cancer $(17,27,28)$. miR-20a overexpression has been observed to inhibit cellular proliferation, invasion and tumor metastasis in certain cancer cell lines $(18,29)$, suggesting a tumor suppressor role. However, certain studies have shown that miR-20a may promote cellular proliferation in colon adenocarcinoma and glioma $(30,31)$, suggesting that it functions as an onco-miRNA. This difference indicates that the effect of miR-20a dysregulation in different types of cancer depends on the specific cell type and extracellular factors. However, few reports exist concerning the potential function of this miRNA in human NSCLC progression. The present study is concerned with the role of miR-20a in the malignant progression of NSCLC cells.

The present study detected miR-20a expression in 35 pairs of human NSCLC and matched normal tissues, as well as in different cell lines, as determined by RT-qPCR. miR-20a was frequently upregulated in tissue specimens and malignant cells. Prior to the current study, however, the role of miR-20a and its target genes in NSCLC was unclear. miR-20a may be a novel tumor oncogene and its deregulation may be associated with the progression of human NSCLC. Therefore, the functions and molecular mechanisms of miR-20a in human NSCLC were the focal point of the present study.

Previous studies have indicated that miR-20a is associated with cellular proliferation (29) and apoptosis (32). In the present study, miR-20a was found to be associated with cell growth, and its knockdown could inhibit the growth of A-549 cells. A soft-agar colony formation assay revealed that miR-20a could promote the growth of A-549 cells. An invasion assay showed that miR-20a was associated with invasion activity in NSCLC 

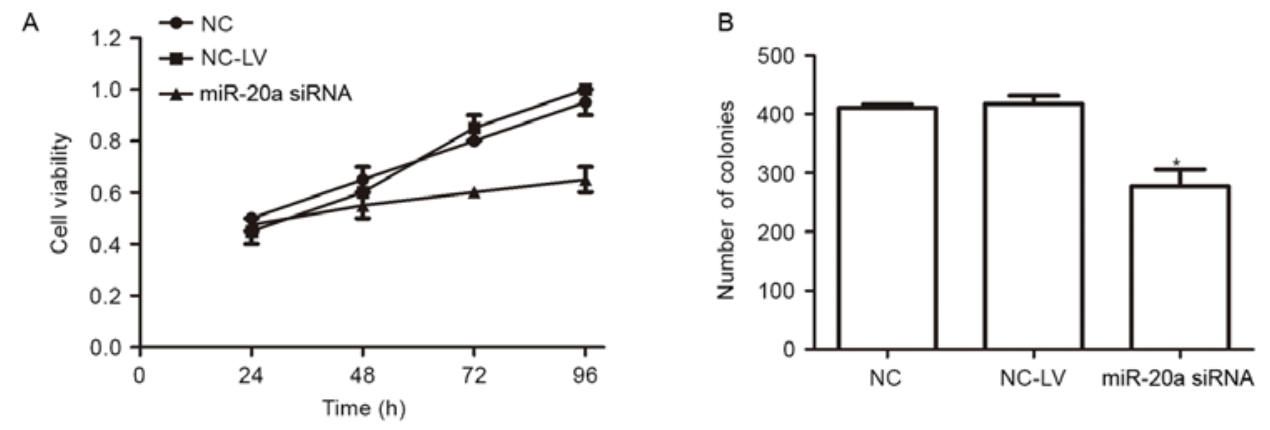

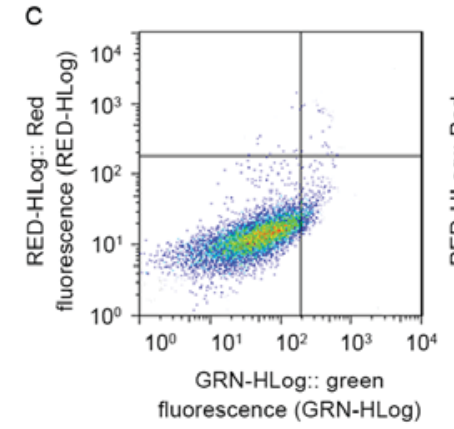

NC

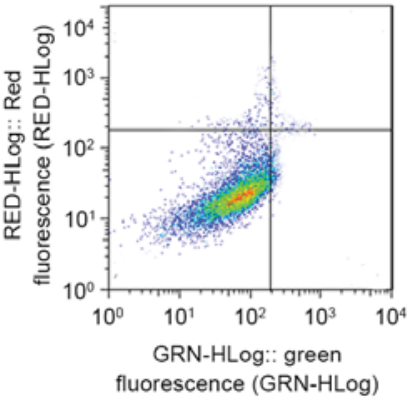

NC-LV

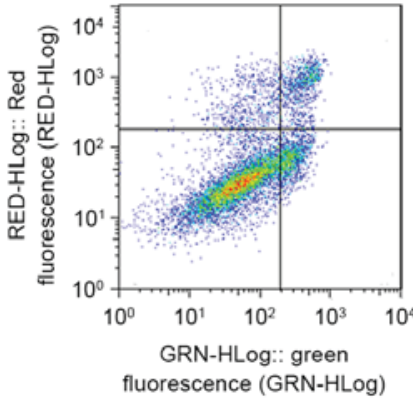

miR-20a siRNA

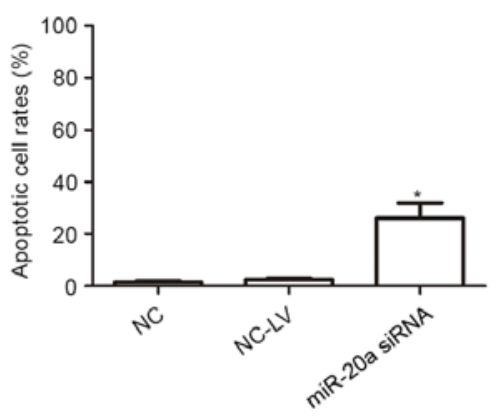

Figure 3. Effect of miR-20a on cell proliferation and apoptosis in A-549 cells. (A) Cell proliferation (CCK-8) assay. A-549 cells were grown and transiently transfected with miR-20a siRNA lentivirus or negative control, then subjected to CCK-8 assay. (B) Representative results of a colony formation assay in A-549 cells transfected with miR-20a inhibitor or the corresponding control. (C) Flow cytometry tumor cell apoptosis assay. Cells were grown and transiently transfected with miR-20a siRNA or the corresponding negative control, then subjected to flow cytometry analysis to assess cell apoptosis. * $\mathrm{P}<0.05$ vs. control groups. miR-20a, microRNA-20a; CCK-8, Cell Counting Kit-8; siRNA, small interfering RNA; NC-LV, A-549 cells transfected with empty lentivirus negative control.
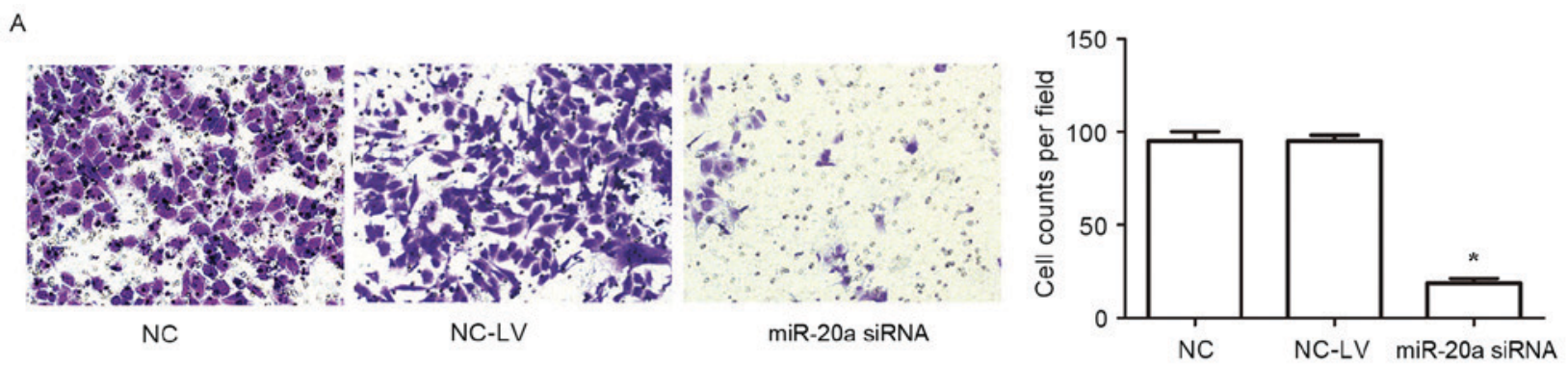

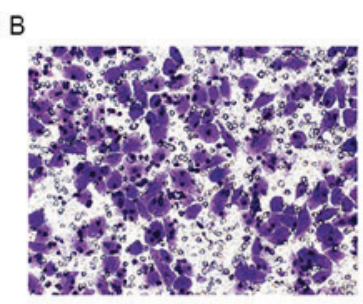

NC

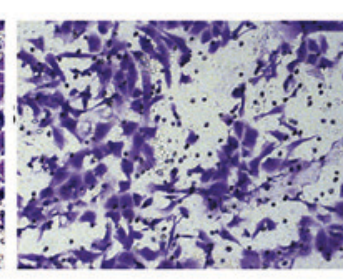

NC-LV

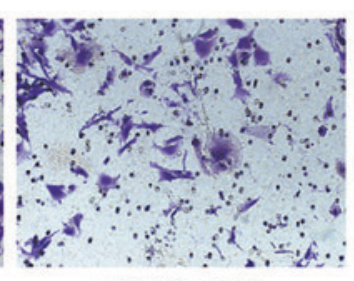

miR-20a siRNA

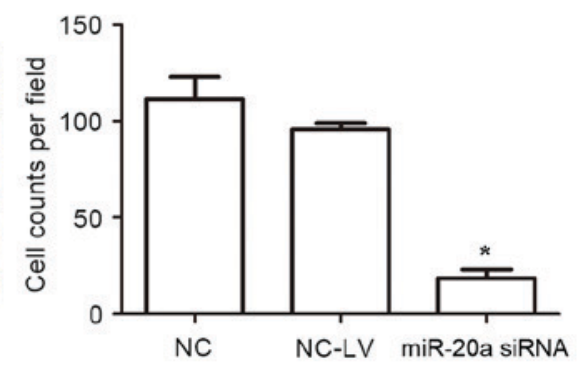

Figure 4. Effects of miR-20a on migration and invasion in NSCLC cells. Three cell lines were grown and transiently transfected with miR-20a siRNA or the corresponding control, then subjected to migration or invasion assay. (A) Representative microscopy images of migratory cells from the non-treated blank group, negative control group, and miR-20a-knockdown group (magnification, x100); (B) Knockdown of miR-20a inhibited invasion of A-549 cells. Crystal violet was used to stain the cells. ${ }^{*} \mathrm{P}<0.05$ vs. controls. miR-20a, microRNA-20a; NC-LV, negative control-empty lentiviral vector.

cells. To investigate the role of miR-20a in NSCLC in vivo, anmiR-20a-knockdown model of NSCLC xenografts in nude mice was generated. Growth curve results demonstrated that miR-20a-knockdown can significantly suppress the growth of NSCLC xenografts in nude mice.

To determine how miR-20a acts as an oncogene further, potential targets were identified using bioinformatic analytic tools. The tumor suppressor EGR2 was downregulated in NSCLC. Luciferase activity assay suggested direct targeting of EGR2 by miR-20a. mRNA and protein levels of EGR2 were decreased in A-549 cells transfected with miR-20a, but were increased in miR-20a-knockdown A-549 cells. These results indicated that EGR2 is a target of miR-20a in NSCLC cells. EGR2 belongs to a multi-gene family that encodes C2H2-type 
A

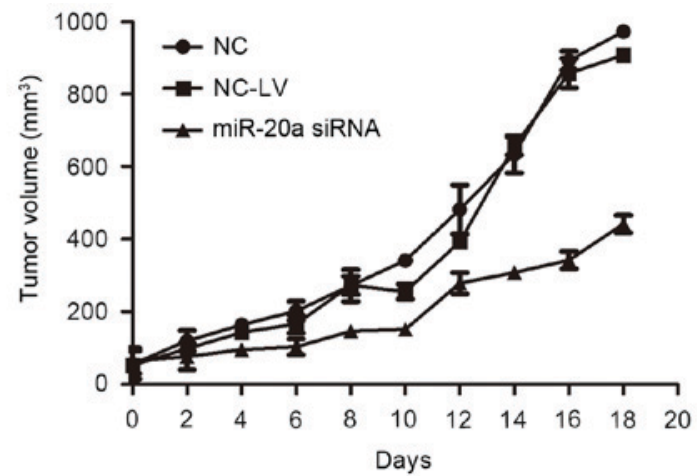

B

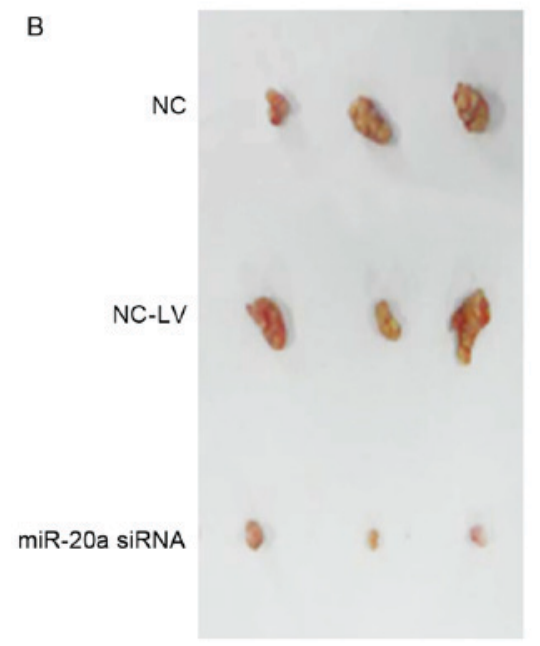

C

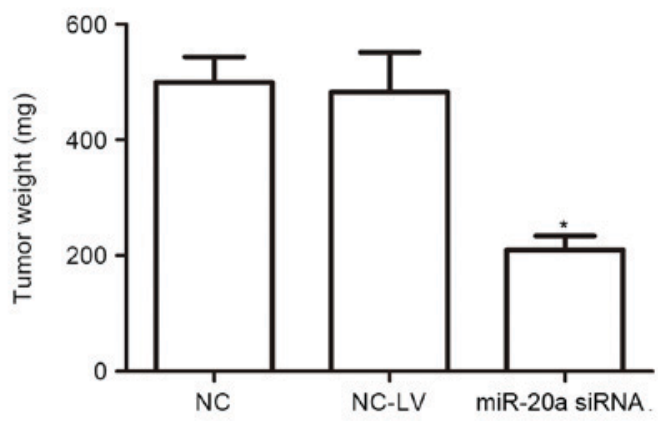

Figure 5. Suppression of tumor growth by miR-20a in the xenograft mouse model. A total of 9 female BALB/c-nu mice were randomly separated into three groups of 3 mice. A-549 cells were transfected with miR-20a siRNA or the corresponding negative control, then injected into left flanks of mice. (A) Tumor growth was measured every 2 days after injection. (B) Images and (C) weights of xenograft tumors. Tumors were harvested on day 18 and weighed. ${ }^{*} \mathrm{P}<0.05$ vs. controls. miR-20a, microRNA-20a; siRNA, small interfering RNA; NC-LV, negative control-empty lentiviral vector.

A

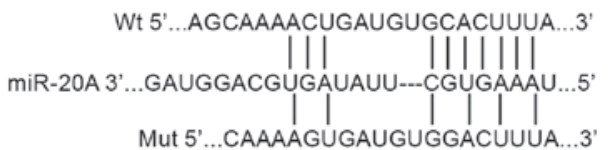

C
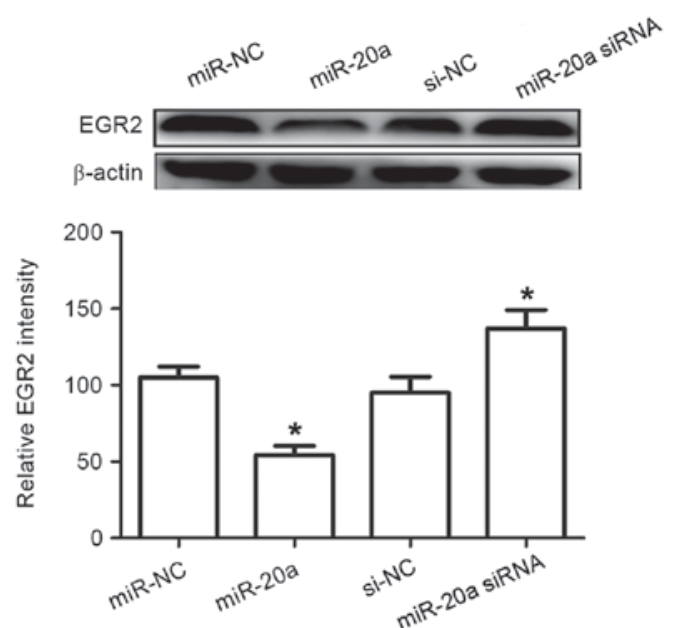

B

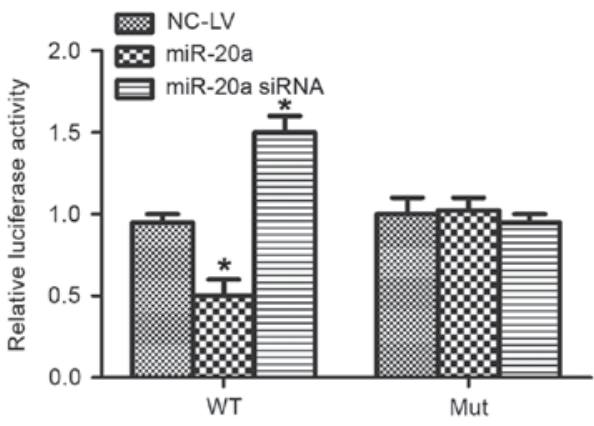

D

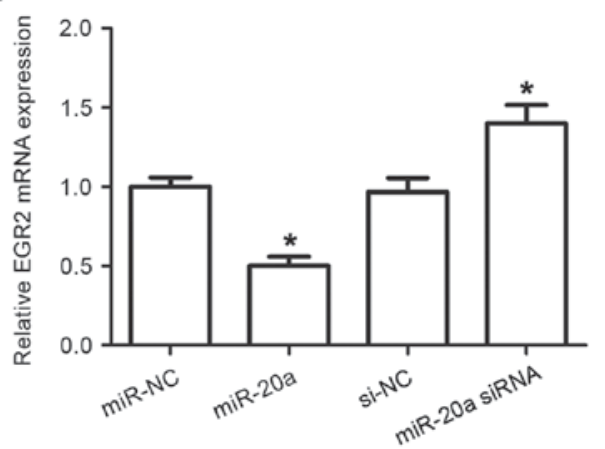

Figure 6. Identification of EGR2 as a target of miR-20a. (A) Wild-type and mutated sequences of the EGR2 3'-UTR (nucleotides 458-465). (B) Luciferase activity was detected once A-549 cells had been co-transfected with negative control, miR-20a precursor or miR-20a siRNA with EGR2 3'-UTR fragment containing either the miR-20a target sequence (WT) or a mutant. (C) Western blot analysis was used to detect EGR2 protein level in cells transfected with miR-20a precursor, siRNA or the corresponding control. (D) Reverse transcription-quantitative polymerase chain reaction was used to detect expression of EGR2 mRNA in cells transfected with miR-20a precursor, siRNA or the corresponding control. $\beta$-actin was used as an internal control. "P<0.05 compared with control. EGR2, early growth response 2; miR-20a, microRNA-20a; WT, wild-type; Mut, mutant; siRNA, small interfering RNA; 3'-UTR, 3'-untranslated region; NC, negative control. 
zinc-finger proteins (33). EGR2 is a key regulator of cellular proliferation and the cell cycle (34). EGR2 is a target of the tumor protein p53 family, and overexpression of EGR2 leads to apoptosis, whereas downregulation of EGR2 attenuates p53-mediated apoptosis (35). EGR2 can be also regulated by other miRNAs (36). The present study sheds fresh light on the regulation of EGR2.

In conclusion, the present study demonstrated that miR-20a expression is upregulated in NSCLC tissues and cells. miR-20a promotes cell growth, migration and invasion by targeting EGR2 in human NSCLC cells. These results suggest that miR-20a serves a critical role in NSCLC growth by regulating EGR2 and indicate that miR-20a could represent a biomarker and therapeutic target in NSCLC therapy.

\section{References}

1. Siegel R, Naishadham D and Jemal A: Cancer statistics 2013. CA Cancer J Clin 63: 11-30, 2013.

2. Siegel RL, Miller KD and Jemal A: Cancer statistics 2015. CA Cancer J Clin 65: 5-29, 2015.

3. Laskin JJ and Sandler AB: State of the art in therapy for non-small cell lung cancer. Cancer Invest 23: 427-442, 2005.

4. Ferguson MK: Diagnosing and staging of non-small cell lung cancer. Hematol Oncol Clin North Am 4: 1053-1068, 1990.

5. Del Vescovo V, Grasso M, Barbareschi M and Denti MA MicroRNAs as lung cancer biomarkers. World J Clin Oncol 5: 604-620, 2014

6. Bartel DP: MicroRNAs: Genomics, biogenesis, mechanism and function. Cell 116: 281-297, 2004.

7. Schickel R, Boyerinas B, Park SM and Peter ME: MicroRNAs: Key players in the immune system, differentiation, tumorigenesis and cell death. Oncogene 27: 5959-5974, 2008.

8. Yang T, Thakur A, Chen T, Yang L, Lei G, Liang Y, Zhang S, Ren $\mathrm{H}$ and Chen M: MicroRNA-15a induces cell apoptosis and inhibits metastasis by targeting BCL2L2 in non-small cell lung cancer. Tumour Biol 36: 4357-4365, 2015.

9. Chen CZ and Lodish HF: MicroRNAs as regulators of mammalian hematopoiesis. Semin Immunol 17: 155-165, 2005.

10. Calin GA and Croce CM: MicroRNA signatures in human cancers. Nature Rev Cancer 6: 857-866, 2006.

11. Li J, Wang Y, Song Y, Fu Z and Yu W: miR-27a regulates cisplatin resistance and metastasis by targeting RKIP in human lung adenocarcinoma cells. Mol Cancer 13: 193, 2014

12. Xiong S, Zheng Y, Jiang P, Liu R, Liu X and Chu Y: MicroRNA-7 inhibits the growth of human non-small cell lung cancer A-549 cells through targeting BCL-2. Int J Biol Sci 7: 805-814, 2011.

13. Guan P, Yin Z, Li X, Wu W and Zhou B: Meta-analysis of human lung cancer microRNA expression profiling studies comparing cancer tissues with normal tissues. J Exp Clin Cancer Res 31: $54,2012$.

14. Tsuchida A, Ohno S, Wu W, Borjigin N, Fujita K, Aoki T, Ueda S, Takanashi M and Kuroda M: miR-92 is a key oncogenic component of the miR-17-92 cluster in colon cancer. Cancer Sci 102: 2264-2271, 2011.

15. Yoshino H, Seki N, Itesako T, Chiyomaru T, Nakagawa M and Enokida H: Aberrant expression of microRNAs in bladder cancer. Nat Rev Urol 10: 396-404, 2013.

16. Li X, Pan JH, Song B, Xiong EQ, Chen ZW, Zhou ZS and Su YP: Suppression of CX43 expression by miR-20a in the progression of human prostate cancer. Cancer Biol Ther 13: 890-898, 2012.

17. Chang CC, Yang YJ, Li YJ, Chen ST, Lin BR, Wu TS, Lin SK Kuo MY and Tan CT: MicroRNA-17/20a functions to inhibit cell migration and can be used a prognostic marker in oral squamous cell carcinoma. Oral Oncol 49: 923-931, 2013.

18. Fan MQ, Huang CB, Gu Y, Xiao Y, Sheng JX and Zhong L: Decrease expression of microRNA-20a promotes cancer cell proliferation and predicts poor survival of hepatocellular carcinoma. J Exp Clin Cancer Res 32: 21, 2013.
19. Xia Y, Chen Q, Zhong Z, Xu C, Wu C, Liu B and Chen Y: Down-regulation of miR-30c promotes the invasion of non-small cell lung cancer by targeting MTA1. Cell Physiol Biochem 32: 476-485, 2013

20. Zhang H, Zhang H, Zhao M, Lv Z, Zhang X, Qin X, Wang H, Wang S, Su J, Lv X, et al: MiR-138 inhibits tumor growth through repression of EZH2 in non-small cell lung cancer. Cell Physiol Biochem 31: 56-65, 2013

21. Wu N, Zhang C, Bai C, Han YP and Li Q: MiR-4782-3p inhibited non-small cell lung cancer growth via USP14. Cell Physiol Biochem 33: 457-467, 2014

22. Livak KJ and Schmittgen TD: Analysis of relative gene expression data using real-time quantitative PCR and the 2(-Delta Delta C(T)) method. Methods 25: 402-408, 2001.

23. Agarwal V, Bell GW, Nam JW and Bartel DP: Predicting effective microRNA target sites in mammalian mRNAs. ELife 4: 10, 2015.

24. Betel D, Wilson M, Gabow A, Marks DS and Sander C: The microRNA.org resource: Targets and expression. Nucleic Acids Res 36: D149-D153, 2008.

25. Wong N and Wang X: miRDB: An online resource for microRNA target prediction and functional annotations. Nucleic Acids Res 43 (Database issue): D146-D152, 2015.

26. Cho WC: OncomiRs: The discovery and progress of microRNAs in cancers. Mol Cancer 6: 60, 2007.

27. Chang Y, Liu C, Yang J, Liu G, Feng F, Tang J, Hu L, Li L, Jiang F, Chen C, et al: MiR-20a triggers metastasis of gallbladder carcinoma. J Hepatol 59: 518-527, 2013.

28. O'Donnell KA, Wentzel EA, Zeller KI, Dang CV and Mendell JT: c-Myc-regulated microRNAs modulate E2F1 expression. Nature 435: 839-843, 2005

29. Zhou J, Liu R, Luo C, Zhou X, Xia K, Chen X, Zhou M, Zou Q, Cao P and Cao K: MiR-20a inhibits cutaneous squamous cell carcinoma metastasis and proliferation by directly targeting LIMK1. Cancer Biol Ther 15: 1340-1349, 2014.

30. Schetter AJ, Leung SY, Sohn JJ, Zanetti KA, Bowman ED, Yanaihara N, Yuen ST, Chan TL, Kwong DL, Au GK, et al: MicroRNA expression profiles associated with prognosis and therapeutic outcome in colon adenocarcinoma. JAMA 299: 425-436, 2008

31. Malzkorn B, Wolter M, Liesenberg F, Grzendowski M, Stühler K, Meyer HE and Reifenberger G: Identification and functional characterization of microRNAs involved in the malignant progression of gliomas. Brain Pathol 20: 539-550, 2010.

32. Zhang G, Liu X, Wang W, Cai Y, Li S, Chen Q, Liao M, Zhang M, Zeng G, Zhou B, et al: Down-regulation of miR-20a-5p triggers cell apoptosis to facilitate mycobacterial clearance through targeting JNK2 in human macrophages. Cell Cycle 15: 2527-2538, 2016

33. Joseph LJ, le Beau MM, Jamieson GA Jr, Acharya S, Shows TB, Rowley JD and Sukhatme VP: Molecular cloning, sequencing and mapping of EGR2, a human early growth response gene encoding a protein with 'zinc-binding finger' structure. Proc Natl Acad Sci USA 85: 7164-7168, 1988.

34. Dzialo-Hatton R, Milbrandt J, Hockett RD Jr and Weaver CT: Differential expression of Fas ligand in Th1 and Th2 cells is regulated by early growth response gene and NF-AT family members. J Immunol 166: 4534-4542, 2001.

35. Unoki M and Nakamura Y: Growth-suppressive effects of BPOZ and EGR2, two genes involved in the PTEN signaling pathway. Oncogene 20: 4457-4465, 2001.

36. Wu Q, Jin H, Yang Z, Luo G, Lu Y, Li K, Ren G, Su T, Pan Y, Feng B, et al: miR-150 promotes gastric cancer proliferation by negatively regulating the pro-apoptotic gene EGR2. Biochem Biophys Res Commun 392: 340-345, 2010.

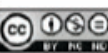

This work is licensed under a Creative Commons Attribution-NonCommercial-NoDerivatives 4.0 International (CC BY-NC-ND 4.0) License. 